\title{
Radiation model of a xenon flash lamp in a laser amplifier pump cavity
}

\author{
Yongzhong $\mathrm{Wu}^{1,2}$, Jianqiang $\mathrm{Zhu}^{1,2}$, Zhixiang Zhang ${ }^{1,2}$, and Yangshuai $\mathrm{Li}^{1,2}$ \\ ${ }^{1}$ National Laboratory on High Power Laser and Physics, Shanghai Institute of Optics and Fine Mechanics, \\ Chinese Academy of Sciences, Shanghai 201800, China \\ ${ }^{2}$ University of Chinese Academy of Sciences, Beijing 100049, China \\ (Received 10 August 2015; revised 2 November 2015; accepted 26 November 2015)
}

\begin{abstract}
Understanding the radiation model of a flash lamp is essential for the reflector design of a laser amplifier. Reflector design often involves several simplifying assumptions, like a point or Lambertian source; either of these assumptions may lead to significant errors in the output distribution. In practice, source non-idealities usually result in sacrificing the amplifier's gain coefficient. We propose a novel test technique for attaining the xenon flash lamp absolute spectral intensity at various angles of view, and then accurately predict radiation distributions and generate the reflector shape. It is shown that due to the absorption of emitted radiation by the lamp itself, the behavior of the radiation model at various wavelengths is different. Numerical results of xenon plasma absorption coefficient were compared with the measured data. A reasonable agreement was obtained for the absorption coefficient parameters. Thus, this work provides a useful analytical tool for the engineering design of laser amplifier reflectors using xenon flash lamps as pumps.
\end{abstract}

Keywords: absorption coefficient; current density; radiation model; xenon flash lamp; xenon plasma

\section{Introduction}

To attain ignition conditions, a laser driver for inertial confinement fusion (ICF) should have output energy of the order of megajoules at the appropriate wavelength over a pulselength of a few nanoseconds ${ }^{[1-4]}$. The rod and disk amplifiers for high-peak-power laser applications are the key components of Nd:glass ICF drivers, which provide the maximum laser energy and are pumped by flash lamps ${ }^{[5,6]}$. Generally, the efficiency of the laser driver depends on the collection efficiency of the amplifier reflectors used to couple the flash lamp light to the Nd:glass ${ }^{[7-10]}$. Reflector design can minimize power and energy requirements of a flash lamp and increase laser efficiency and lamp life. However, no model has been developed that can adequately describe the radiation style of a flash lamp.

Two theoretical descriptions of the radiation model are presently available in the literature ${ }^{[11]}$. The first theory of the imaging reflector is based on the pumping system of a reflecting linear ellipse with flash lamps and Nd:glass at the foci. Here, rays are considered to leave normal to the surface of the lamp tube; this theory tends to regard the flash lamp as a point source that forms a cylindrical illuminant.

Correspondence to: Yongzhong Wu, No. 390, Qinghe road, Jiading, Shanghai 201800, China. Email: wuyongzhongtmac@126.com
The second theory treats the xenon plasma as a graybody radiator, which can emit as well as absorb pump light. The radiating xenon plasma is optically thick, meaning that pump light emanates from the surface of the plasma and not from within its bulk. The absorption of emitted radiation by xenon plasma itself is relatively sensitive to the flash lamp parameters.

In this study, we assume that xenon plasma absorption coefficients change with current density, lamp diameter, and xenon pressure. Our key purpose is to provide an exact flash lamp radiation model for reflector design. The accuracy of the model is demonstrated by comparison with experimental data of absolute spectral-intensity measurements at one point on the surface of a lamp tube.

\section{Calculation results}

\subsection{Physical description}

Following Trenholme-Emmett ${ }^{[12]}$, we discovered that the radiation model was a uniform cylinder of hot plasma that filled or nearly filled the lamp bore, and the output spectral distribution of the flash lamp operating in the quasi-stationary regime was based on a large quantity of 
experimental data. These data were useful for the accurate prediction of radiant output over a wide range of parameters. Kirchhoff's law ${ }^{[13]}$ stated that if the plasma absorption coefficient at wavelength $\lambda$ was given by $\alpha(\lambda)$, then the emission intensity as viewed through a uniform plasma at temperature $T$ and over a length $l$ was given by

$$
I(\lambda, T)=[1-\exp (-\alpha(\lambda) l)] * I_{\mathrm{bb}}(\lambda, T),
$$

where $I_{\mathrm{bb}}(\lambda, T)$ was the blackbody intensity at temperature $T$.

The emissivity was defined as

$$
\varepsilon_{\lambda}=1-\exp (-\alpha(\lambda) l)
$$

The flash lamp radiation was made up of both line and continuum emission, similar to the absorption coefficient $\alpha(\lambda)$, and could be approximated by

$$
\begin{aligned}
\alpha(\lambda)= & {\left[\alpha_{c}(\lambda)+\alpha_{l}(\lambda)\right] * M(i, d, p), } \\
\alpha_{c}(\lambda)= & \alpha_{1} \exp \left\{-\left[\left(\lambda-\lambda_{1}\right) / \omega_{1}\right]^{2}\right\} \\
& +\alpha_{2} \exp \left\{-\left[\left(\lambda-\lambda_{2}\right) / \omega_{2}\right]^{2}\right\}, \\
M(i, d, p)= & \exp \left[1.57 \ln (i)+0.071 \ln ^{2}(i)\right. \\
& +0.19 \ln (d)+0.46 \ln (p)],
\end{aligned}
$$

where $\alpha_{c}(\lambda)$ was the continuum absorption coefficient; $\alpha_{1}=$ $2.37 \times 10^{-2} \mathrm{~cm}^{-1}$ and $\alpha_{2}=1.5 \times 10^{-3} \mathrm{~cm}^{-1}$ were two fitting coefficients; $\lambda_{1}=0.7 \mu \mathrm{m}$ and $\lambda_{2}=0.3 \mu \mathrm{m}$ were the central wavelengths of two Gaussian functions with bandwidths $\omega_{1}=0.33 \mu \mathrm{m}$ and $\omega_{2}=0.14 \mu \mathrm{m}$, respectively. Fitting the line absorption coefficient $\alpha_{l}(\lambda)$ at wavelength $\lambda$ by 20 Lorenz curves with central wavelengths, amplitudes, and line width was first implemented by Powell ${ }^{[14]} . M(i, d, p)$ was the standardization factor, $i$ was the current density, $d$ was the lamp diameter, and $p$ was the xenon pressure.

Given the relationship between the plasma temperature and the physical characteristics of the lamp, fitting the temperature function ${ }^{[15,16]}$ to the experimental results, we have

$$
T=9300 i^{0.18} d^{0.092} p^{-0.017}
$$

Then, having obtained the temperature for a given input electrical power from Equation (6), Equation (3) was used to calculate the absorption coefficient. Finally the radiation spectrum power would be calculated from Equation (1).

Flash lamps are filled with xenon and have variations in the bore diameter, constant 114-cm arc lengths, and walls made of cerium-doped quartz. A simplified equivalent drive circuit with two lamps connected in series is illustrated in Figure 1. This circuit has a capacitance of $125 \mu \mathrm{F}$ and an inductance of $250 \mu \mathrm{H}$.



Figure 1. Schematic diagram of the electrical circuit

\subsection{Calculated result}

The absorption coefficients are presented in Figure 2(a) for $1.5-\mathrm{cm}$-bore flash lamps with a current density of 2.5$3.5 \mathrm{kA} / \mathrm{cm}^{2}$. The shape of these curves reflects the flash lamp efficiency in producing light in the Nd:glass pump region (wavelengths between 400 and $1000 \mathrm{~nm}$ ). They also show that flash lamp opacity is a function of current density and wavelength. For a wavelength $\lambda$ less than $800 \mathrm{~nm}$, the absorption coefficient rises linearly. For $800 \mathrm{~nm}<$ $\lambda<850 \mathrm{~nm}$, the absorption coefficient is a downward parabola, whereas for a wavelength $\lambda$ longer than $850 \mathrm{~nm}$, the absorption coefficient noisily reduces. Figure 2(b) further illustrates how a larger current density provides a much better characterization of wavelength intensity. Figure 2(c) shows the lamp emissivity $(\lambda=700 \mathrm{~nm})$ versus plasma length at three lamp current densities. We interpreted the general shape of these curves to be caused by those reasons discussed for Figure 2(a). Figure 2(d) shows the dependence of the calculated emissivity on plasma length for three wavelengths. For the radiation transport in plasma longer than the lamp radius, the plasma is almost opaque when the wavelength is longer than $800 \mathrm{~nm}$ and the lamp radiation model is a hollow cylinder. For the radiation transport in plasma in a lamp bore, radiation will fill the lamp bore when the wavelength is shorter than $800 \mathrm{~nm}$. Flash lamp radiation efficiency ${ }^{[17,18]}$ is defined as the radiant spectrum power divided by the electrical input energy $p_{\text {in }}$ :

$$
\eta=\frac{\iint I(\lambda) \mathrm{d} \lambda \mathrm{d} t}{p_{\text {in }}} .
$$

According to Equation (7) and Figure 2(e), the observed decrease of radiation efficiency with increasing flash lamp current density is caused by a flash lamp opacity mechanism in which some of the light absorbed by the xenon plasma is not reradiated in the pump region.

For the circuit, we can represent the system using the following equation ${ }^{[19-21]}$ :

$$
\begin{gathered}
L \frac{\mathrm{d} i}{\mathrm{~d} t}+K_{0}|i|^{1 / 2}+\frac{1}{c} \int_{0}^{t} i \mathrm{~d} t+R i=V_{0} \\
K_{0}=1.3 \frac{s}{d}\left(\frac{P}{450}\right)^{0.2}
\end{gathered}
$$



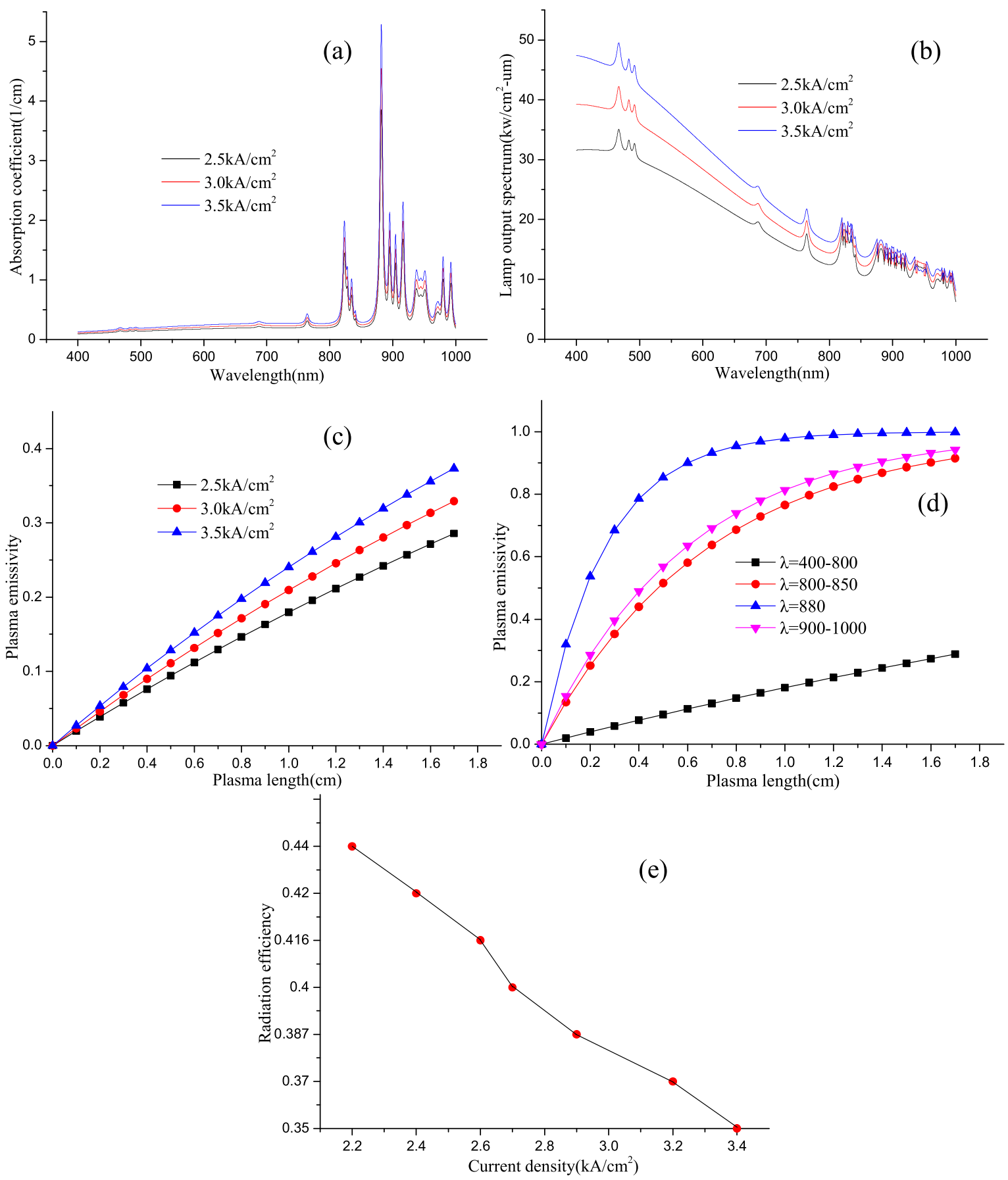

Figure 2. Effect of current density on the xenon flash lamp absorption coefficient: (a) absorption coefficient; (b) lamp output spectrum; (c) plasma emissivity at different current densities with the same wavelength; (d) plasma emissivity at different wavelengths with the same current density; (e) relationship between current density and radiation efficiency.

where $V_{0}$ is the initial voltage on the capacitor, $L$ is the inductance, $C$ is the bank capacitor and $S$ is the total arc length.

We chose the equivalent current densities for the flash lamp bore from 0.8 to $2 \mathrm{~cm}$. The electrical time dependences of the lamp current pulses were calculated by Equation (8). As shown in Figure 3(a), the lamp with 2-cm-bore current pulse peaks later than all other lamps. In addition, note that the peak time of current density is a function of bore diameter. This is likely caused by the arc expansion due to the plasma's diffusion velocity and temperature gradient from the lamp centerline to the shell, as shown in Figure 3(b). We already know that the value of $M(i, d, p)$ is determined by lamp parameters and current density plays a leading role. 

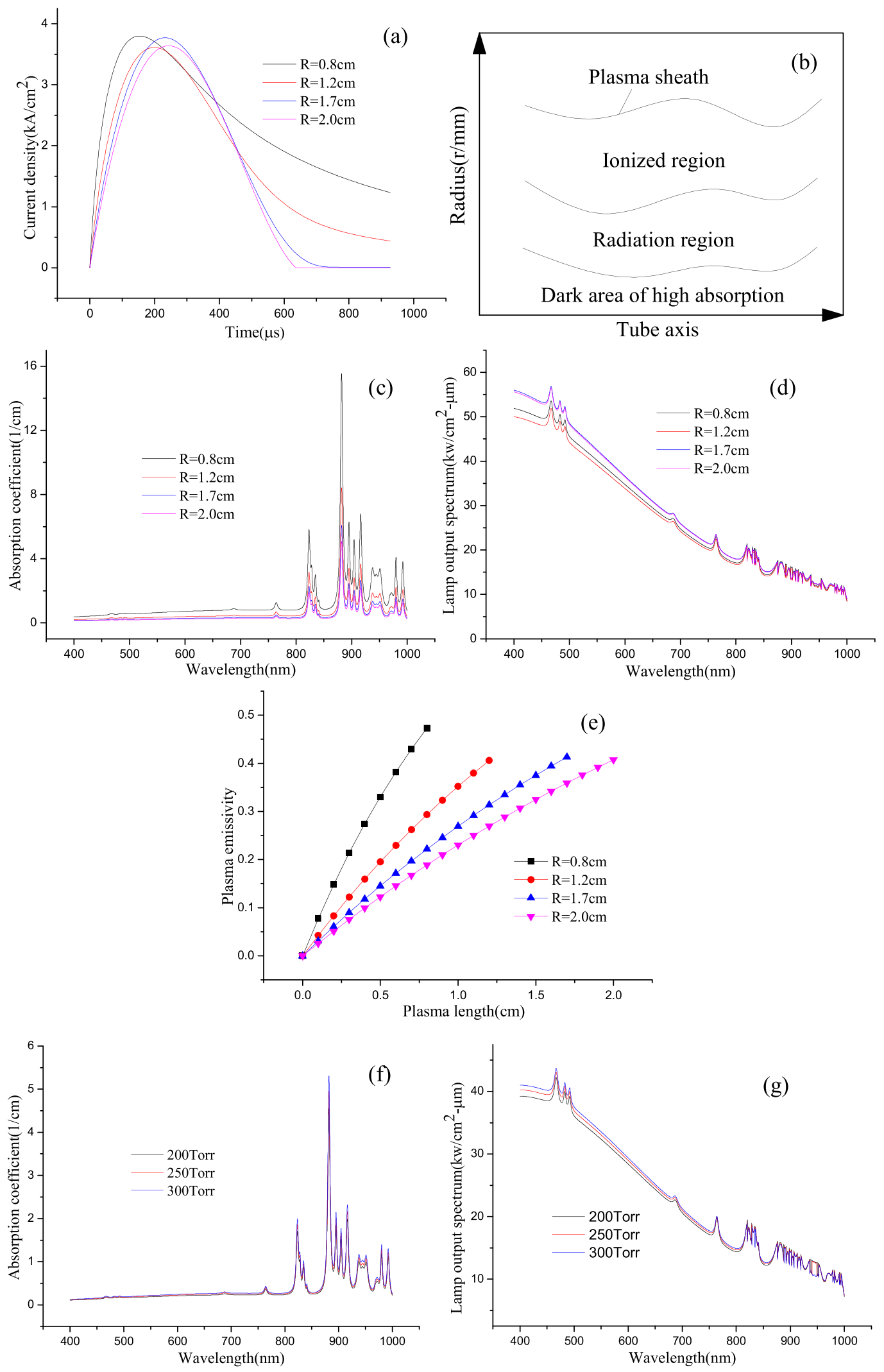

Figure 3. Effect of lamp diameter and xenon pressure on the absorption coefficient: (a) current pulse shapes at different xenon lamp diameters; (b) schematic diagram of xenon plasma; (c) absorption coefficient; (d) lamp output spectrum; (e) plasma emissivity for $\lambda=700 \mathrm{~nm}$ at different xenon lamp diameters; (f) absorption coefficient at different xenon pressures; (g) lamp output spectrum. 


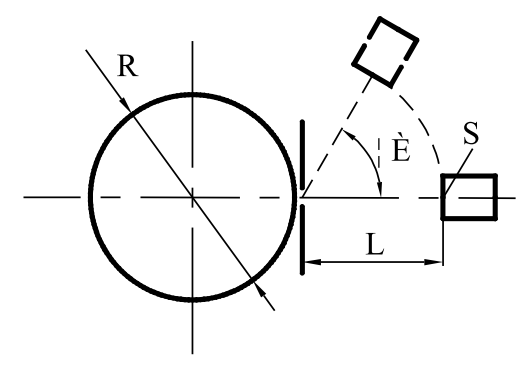

Figure 4. Schematic diagram of the test facility.

It is evident from Equations (3) and (5) that there is little to be decreased in flash lamp absorption coefficient and wavelength intensity by varying the bore diameter, at least over the $0.8-2 \mathrm{~cm}$ range. After careful inspection of the curves in Figures 3(c) and 3(d), we believe that the slight difference in absorption coefficient is caused by mistaking the thermal conduction to the tube for the energy of reabsorbed plasma. Analysis of Figure 3(e) shows that the emissivity of the plasma is quite closely approximate to the performance of the absorption coefficient and spectral output. An empirical description of the voltage-current relationship of the lamp is given by ${ }^{[12]}$

$$
V_{0}=k_{0} i^{0.5}
$$

and the total electrical input energy from the relationship is $^{[12]}$

$$
E_{0}=\frac{1}{2} C V_{0}^{2} \text {. }
$$

In lamps at the same current densities, if the bore diameter decreases, $k_{0}$ and $V_{0}$ increase. Since the radiant spectrum powers are almost the same, we can meet the output energy requirement through electrical input energy with a smaller bore diameter. Analogous to the bore diameter results, the xenon pressures have a similar effect on the radiation, as shown in Figures 3(f) and 3(g).

\section{Experimental verification}

\subsection{Experimental method}

The radiated flash lamp energy depends on the depth of the xenon plasma viewed, and therefore not only on the lamp dimensions, but also on its orientation. Figure 4 shows a schematic of the setup used for the absolute spectralintensity measurements at various angles of view. One flash lamp was vertically fixed and masked by non-reflection material to make a small tube area point, and the photomultiplier was mounted on a turntable with a fixed distance of its detective area from the lamp axis of $L$. The other lamp was placed in a black box and it was ensured that no light radiated outward. Each angle tests three times and takes an average, and the time interval between the two is at least 15 minutes; otherwise the xenon flash lamp would not cool completely. We can calculate the absorption coefficient of the xenon plasma by comparing the energy data at the viewing angles with the data from the normal.

\subsection{Experimental results}

For the 2-cm-bore and 127-cm arc length flash lamp, the circuit has a capacitance of $125 \mu \mathrm{F}$ and an inductance of $250 \mu \mathrm{H}$. A capacitor voltage of $24 \mathrm{kV}$ is used to attain a current pulse amplitude up to $6.2 \mathrm{kA}$. The theoretical prediction of the pulse shape was seen to be in reasonably good agreement with the measurement data as shown in Figures 5(a) and 5(b).

As a check on the reproducibility of test facility, we placed a photomultiplier at a fixed distance from the normal direction of the lamp surface. All test results are nearly identical, which means that the system has good performance stability and there is no visible change in the appearance of facility during the experiment, as shown in Figure 6(a). The exit slit on the surface of the lamp forms a point source to allow light from the lamp to fall directly into the
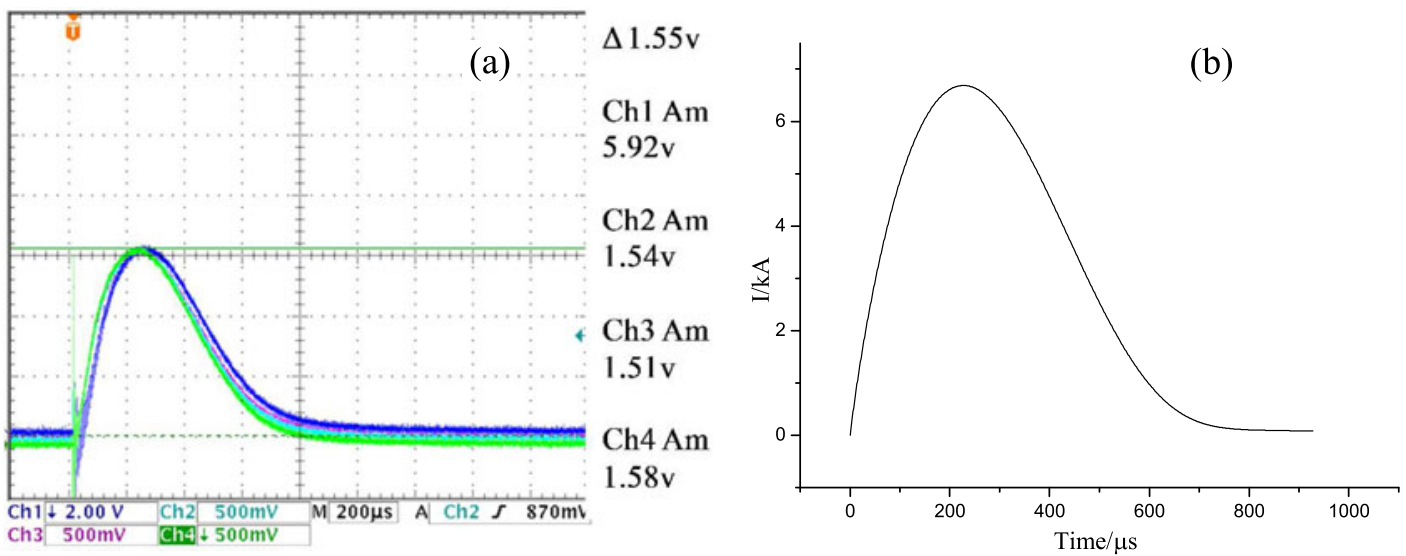

Figure 5. Current pulse shape: (a) experimental result; (b) calculated result. 

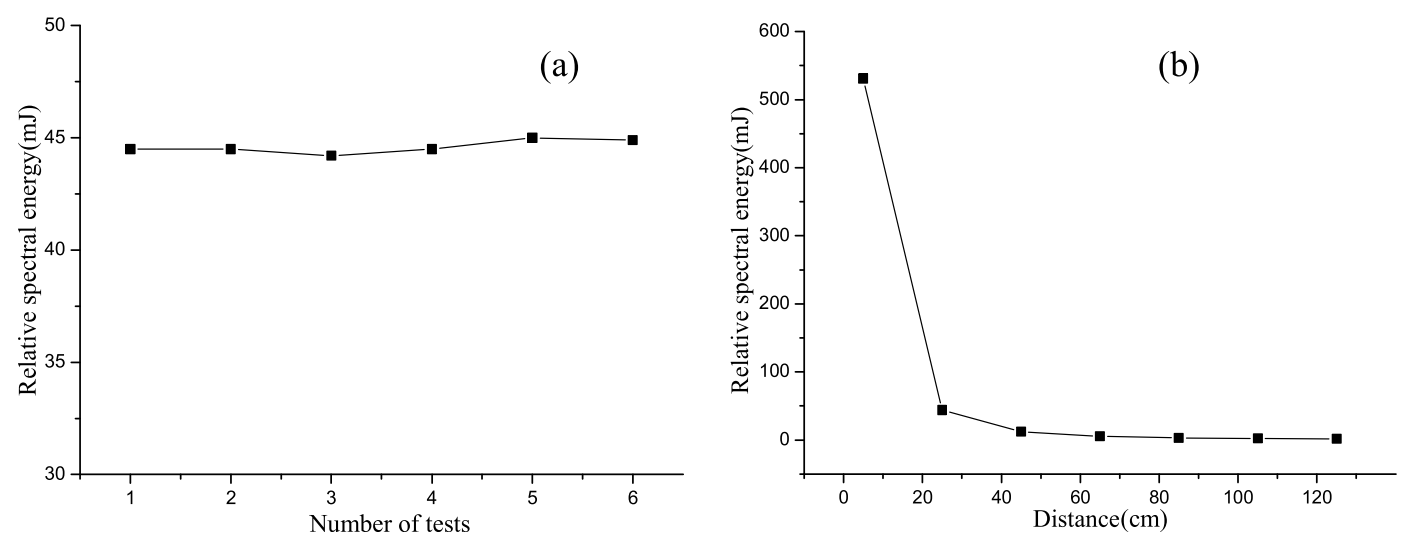

Figure 6. (a) Test system stability; (b) relationship between test distance and emission power.
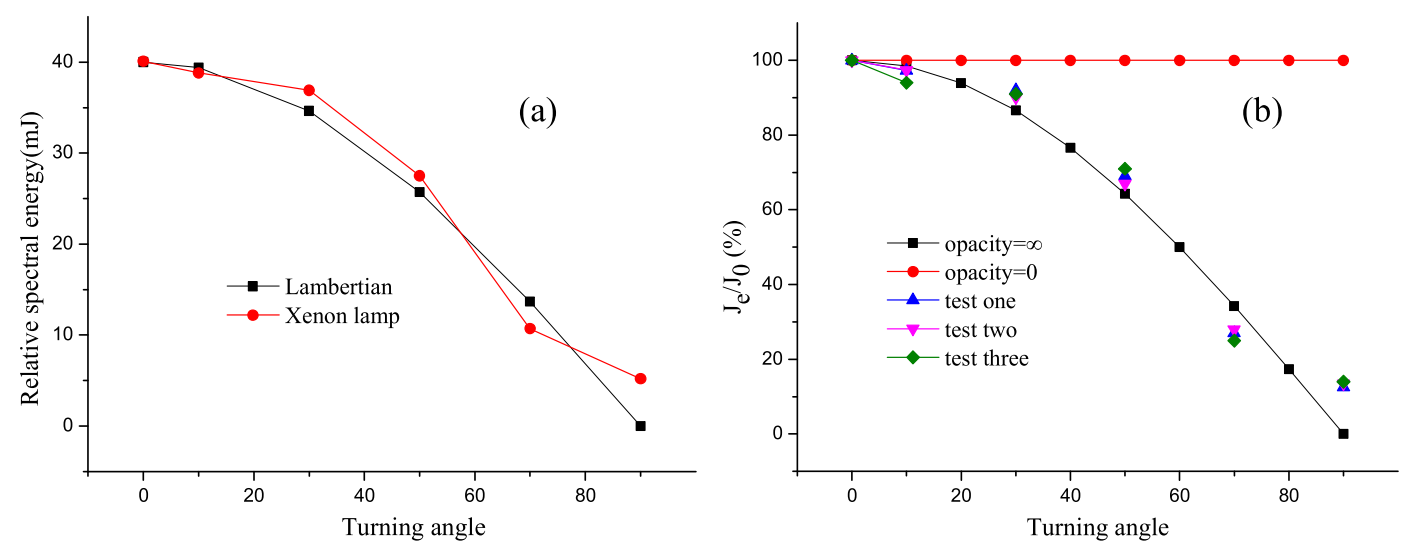

Figure 7. Xenon lamp radiation model: (a) relative spectral power at different angles; (b) power ratio of $J_{\theta} / J_{0}$.

photomultiplier. The solid angle ${ }^{[22]}$ that the photomultiplier subtends at the point source is

$$
\mathrm{d} \Omega=\frac{S}{L^{2}},
$$

where $S$ is the photomultiplier's detection area and $L$ is the distance between the photomultiplier and the lamp. The time-dependent wavelength intensity in the tube normal direction over the current pulse is obtained by

$$
\int I(\lambda) \mathrm{d} t=\int_{0}^{t} J_{\theta=\frac{\pi}{2}} \mathrm{~d} t=\frac{\mathrm{d} e}{\mathrm{~d} \Omega}
$$

where $J_{\theta=\pi / 2}$ is the signal detected by the photomultiplier and $\mathrm{d} e / \mathrm{d} \Omega$ is the wavelength $\lambda$ power emitted by the lamp in the normal direction per unit solid angle over the current pulse. According to Equation (11), the solid angle falls off with increasing distance $L$. As shown in Figure 6(b), when the distance $L$ is shorter than $40 \mathrm{~cm}$ the power sharply decreases. If the distance is longer than $40 \mathrm{~cm}$, the solid angle is smaller than $9 \times 10^{-2}{ }^{\circ} \mathrm{C}$ and we can assume that the photomultiplier's surface area $S$ has no effect on the constant $\mathrm{d} e$. The wavelength power at constant wavelength $\lambda$ and
Table 1. Test results.

\begin{tabular}{lcccccc}
\hline & \multicolumn{6}{c}{ Angle (deg.) } \\
\cline { 2 - 7 } Test & 0 & 10 & 30 & 50 & 70 & 90 \\
\hline Test one $(\mathrm{mJ})$ & 41.5 & 41.3 & 40.2 & 28.6 & 10.89 & 4.93 \\
Test two (mJ) & 39.6 & 37 & 35.8 & 28.05 & 10.07 & 5.51 \\
Test three (mJ) & 39.2 & 38.1 & 34.8 & 25.9 & 11.17 & 5.32 \\
Mean & 40.1 & 38.8 & 36.9 & 27.5 & 10.7 & 5.2 \\
\hline
\end{tabular}

current density is found by integrating Equation (12) will not change, and so do the $J_{\theta=\pi / 2}$.

Power of the wavelength $(532 \mathrm{~nm})$ dependence of the various angles of view (which occurred at the flash lamp peak current density of $3.5 \mathrm{kA} / \mathrm{cm}^{2}$ ) is presented in Table 1 for a $1.7-\mathrm{cm}$-bore lamp at a distance of $40 \mathrm{~cm}$. Figure 7(a) illustrates the wavelength power angular distribution: test results and the power dependence on Lambert's law are simultaneously displayed. In addition, the test results are observed to decrease as the viewing angle increases from 0 to $90^{\circ}$. In general, the lamp emission falls off with the angle approximately as $k_{0} \cos \theta$ (Refs. [23, 24]):

$$
I_{\theta}=K_{0} * I_{0} \cos \theta
$$


where the correction factor of $k_{0}$ is determined by the wavelength. For viewing angle $\theta=90^{\circ}$, the power $I_{\theta}=$ $5.2 \mathrm{~mJ}$ is found to radiate directly from the lamp plasma and not from stray reflections ${ }^{[25]}$. This means that the lamp radiation model is not a point source.

The measured variations of flash lamp absorption coefficient $\alpha(\lambda)$ with wavelength $532 \mathrm{~nm}$ at constant lamp current density and lamp bore are summarized in Figure 7(b). The $y$-axis shows the emission power at an angle with respect to the normal versus the radiation power in the normal ${ }^{[26]}$. For the absorption coefficient $\alpha(\lambda) \approx \infty$, the Lambertian source provides emission light from different positions along the lamp shell. For the absorption coefficient $\alpha(\lambda)=0$, there is no absorption in the plasma. The measurement results of flash lamp opacity are in qualitative agreement with the absorption coefficient $\alpha(\lambda)=3 \mathrm{~cm}^{-1}$ and the calculated results of absorption coefficient $\alpha(\lambda)=2 \mathrm{~cm}^{-1}$. We attribute the measurement error to neglecting the wrapping material thickness. The flash lamp radiation model is shown to be a reasonably accurate predictor of radiant energy at high power.

\section{Conclusion}

The measured and calculated flash lamp radiation models are clearly in good agreement for the case where the lamp is treated as a volume source radiating light outward. It is of interest to estimate the effect that various changes in the lamp parameters would have on the absorption coefficient if all other circuit parameters remained constant. Increasing the lamp current density will increase the absorption coefficient. No dependence of the lamp absorption coefficient on the lamp bore and xenon pressure was observed. For the wavelengths ranging from 400 to $800 \mathrm{~nm}$, the lamp plasma absorption coefficient is so small that the lamp radiated in the whole tube. For wavelengths ranging from 800 to $850 \mathrm{~nm}$, the radiation volume is a hollow cylindrical model. For wavelengths ranging from 850 to $1000 \mathrm{~nm}$, the radiation volume is a thin-shell Lambertian.

\section{Acknowledgments}

The authors would like to acknowledge Dr. Jianqiang Zhu for modifying and proofreading the manuscript. Grants from the Chinese and Israeli cooperation project on high-power laser technology (2010DFB70490) supported this study.

\section{References}

1. S. Yu. Gus'kov, N. V. Zmitrenko, D. V. Il'in, and V. E. Sherman, J. Exp. Theoret. Phys. 119, 958 (2014).

2. R. Florido, R. C. Mancini, T. Nagayama, R. Tommasini, J. A. Delettrez, and S. P. Regan, Phys. Plasmas 21, 102709 (2014).

3. S. X. Hu, V. N. Goncharov, T. R. Boehly, R. L. McCrory, S. Skupsky, L. A. Collins, J. D. Kress, and B. Militzer, Phys. Plasmas 22, 056304 (2015).

4. S. Eliezer and S. V. Pinhasi, High Power Laser Sci. Eng. 1, 44 (2013).

5. V. Bychkov, M. Modestov, and C. K. Law, Progr. Energy Combust. Sci. 47, 32 (2015).

6. C. Tian, J. Chen, B. Zhang, L. Q. Shan, W. M. Zhou, D. X. Liu, B. Bi, F. Zhang, and W. W. Wang, Opt. Express 23, 12362 (2015).

7. S. Jia, R. Li, J. Liu, X. Li, X. Song, and H. Li, Plasma Sci. Technol. 15, 640 (2013).

8. E. H. Baksht, M. Lomaev, A. N. Panchenko, D. V. Rybka, V. F. Tarasenko, M. Krishnan, and J. Thompson, Proc. SPIE 6263, 626316 (2006).

9. R. E. Benchea, I. Cretescu, and S. Kalinowski, Anal. Methods 5, 3650 (2013).

10. H.-J. Kim, D.-J. Kim, J.-T. Hong, G.-C. Xu, and D.-G. Lee, J. Electr. Engng Technol. 6, 275 (2011).

11. G. J. Linford, Appl. Opt. 33, 8333 (1994).

12. J. L. Emmett, A. L. Schawlow, and E. H. Weinberg, J. Appl. Phys. 35, 2601 (1964).

13. H. Powell, A. Erlandson, and K. Jancaitis, Proc. SPIE 609, 78 (1986).

14. H. T. Powell, A. C. Erlandson, K. S. Jancaitis, and E. M. James, Proc. SPIE 1277, 103 (1990).

15. J. F. Holzrichter and J. L. Emmett, Appl. Opt. 8, 1459 (1969).

16. R. H. Dishington, W. R. Hook, and R. P. Hilberg, Appl. Opt. 13, 2300 (1974).

17. R. J. F. Tucker, N. Cochran, and G. L. Morelli, Proc. SPIE 8599, 85990X (2013).

18. E. H. Baksht, M. Lomaev, A. N. Panchenko, D. V. Rybka, V. F. Tarasenko, M. Krishnan, and J. Thompson, Proc. SPIE 6263, 626316 (2006).

19. T. Efthymiopoulos and B. K. Garside, Appl. Opt. 16, 70 (1977).

20. I. S. Marshak, Appl. Opt. 2, 793 (1963).

21. J. G. Edwards, Appl. Opt. 6, 837 (1967).

22. H. Elloumi, G. Zissis, T. Berthier, and J. D. Jean, J. Quant. Spectrosc. Radiat. Transfer 86, 361 (2004).

23. M. A. Gusinow, Appl. Opt. 14, 2645 (1975).

24. W. Lama, T. J. Hammond, and P. J. Walsh, Appl. Opt. 21, 654 (1982).

25. R. Zainal, A. R. Tamuri, Y. M. Daud, and B. Noriah, AIP Conf. Proc. 1250, 133 (2010).

26. D. A. Jacobsen, Proc. SPIE 5524, 295 (2004). 